International Journal of Biomedicine | June 2019 - Volume 9, Issue Suppl_1: Abstracts From the Second Russian International Conference "Cryo-electron microscopy 2019: achievements and prospects"

POSTER ABSTRACT PRESENTATIONS

SESSION TITLE: STRUCTURE AND FUNCTIONS OF THE TRANSCRIPTION AND TRANSLATION APPARATUS OF THE CELL

DOI: 10.21103/IJBM.9.Suppl_1.P16

\title{
Abstract P-16: Interaction of Poly(ADP-Ribose)Polymerase1 and Poly(ADP- Ribose)Polymerase2 with Nucleosome during Base Excision Repair
}

\author{
Mikhail M. Kutuzov ${ }^{1,2}$, Ekaterina A. Belousova ${ }^{1,2}$, Olga I. Lavrik ${ }^{1,2}$, Svetlana N. Khodyreva ${ }^{1}$ \\ ${ }^{1}$ Institute of Chemical Biology and Fundamental Medicine, Novosibirsk, Russia; ${ }^{2}$ Novosibirsk State \\ University, Novosibirsk, Russia
}

Background: The genome is always exposed to different kinds of DNA damaging agents. DNA repair systems in cells are responsible for the genome integrity. The damages, which do not make strong disturbance of double stranded DNA structure, are normally processed by the base excision repair (BER) system. To the moment, this system is well characterized, but the details of regulation are still under investigation. DNA compaction additionally complicates the functioning of repair systems. For successful DNA repair, BER stages, as well as the degree of DNA compaction, should be regulated. Poly(ADPribose)polymerase1 (PARP1) and poly(ADP-ribose)polymerase2 (PARP2) are key BER regulatory members, which are also known to participate in the regulation of chromatin remodeling.

Methods: Polyacrylamide gel electrophoresis.

Results: In our study, we focused on the investigation of the influence of PARPs on the activity of major enzymes of the BER system - APE1 and DNA polymerase beta, using both reconstituted nucleosomes and naked DNAs. We obtained the inhibitory effect of PARP1 and PARP2 on the activity of both APE1 and DNA polymerase beta. This effect was attenuated in the presence of $\mathrm{NAD}^{+}$, under conditions of poly(ADP-ribosyl)ation.

Conclusion: Our results additionally confirm the currently relevant model for the regulation of the interactions of PARP1 and PARP2 with DNA. In particular, under ADP-ribosylation, autopoly(ADPribosyl)ation of these PARPs occurs that contributes to the dissociation of their complexes with the DNA/nucleosome, due to electrostatic repulsion between the DNA and the negatively charged polymer of ADP-ribose, which is covalently attached to PARP. The structural data could clarify the points of interaction and location of PARPs relative to each other or to nucleosome core and BER proteins during repair.

Key Words: nucleosome • DNA-repair • poly(ADP-ribose)polymerase1 • poly(ADP-ribose)polymerase 2 
Sources of Funding: This work was supported by RSF project № 17-74-20075

International Journal of Biomedicine. 2019;9 Suppl 1: S23. doi: 10.21103/IJBM.9.Suppl_1.P16

(C)2019 International Medical Research and Development Corporation 